\section{El proceso de interacción investigadores y tomadores de decisiones: un estudio de caso}

\author{
Interaction between researchers and \\ decision-makers: a case study
}

Ernesto Báscolo 1

Natalia Yavich 1

Adolfo Sánchez de León 2

\footnotetext{
${ }^{1}$ Instituto de la Salud Juan Lazarte, Rosario, Argentina. 2 Seguro Público de Salud, Ministerio de Salud de la Provincia de Buenos Aires, La Plata, Argentina.

Correspondencia E. Báscolo Instituto de la Salud Juan Lazarte.

Pasaje 12 de Octubre 860 (2000) Rosario, Provincia de Santa Fé, Argentina. ebascolo@yahoo.com
}

\begin{abstract}
This article analyzes the process of interaction between the Instituto de la Salud Juan Lazarte research team and the Buenos Aires Public Health Insurance (PHI) management team during the design and development of the study titled "PHI Institutional Capability Analysis and Performance Evaluation", currently underway. From a cross-disciplinary perspective, examining different areas of interaction between the SPS management team and the research team, the relationship is characterized as an application of the "interaction model". This approach promotes the construction of interfaces that allow the development of negotiation and collaboration between the scientific and political "communities". Application of this model has produced changes in the conceptual and methodological framework and in substantive issues during implementation of the SPSMI.
\end{abstract}

Health Insurance; Social Interaction; Health Management

\section{Introducción}

Este artículo analiza el proceso de interacción entre los equipos de investigación (EI) del Instituto de la Salud Juan Lazarte y de gestión (EG) del Seguro Público de Salud (SPS) del Ministerio de Salud de la Provincia de Buenos Aires, Argentina, durante las etapas de diseño y desarrollo del protocolo de investigación de evaluación del SPS.

Esta investigación es producto de una convocatoria que promueve proyectos colaborativos entre EI y EG, a cargo de la implementación de políticas de extensión de la protección social en salud, para mejorar la utilización de los resultados de la investigación en la formulación de políticas de salud. Tuvo una duración de dos años de trabajo, en donde investigadores y tomadores de decisión asumen el compromiso de un trabajo colaborativo, resguardando sus correspondientes autonomías, tanto en el campo académico/científico, como en la aplicación de los resultados.

El presente artículo se estructura del siguiente modo. En primer lugar, se desarrolla el marco conceptual utilizado para el análisis. Seguidamente, se presenta una caracterización del objeto de estudio. Posteriormente, se describe la metodología y el proceso de interacción y sus cambios producidos. A continuación se acompaña una discusión de los resultados e implicancias del proceso de interacción empren- 
dido. Finalmente, se presentan algunas conclusiones preliminares.

\section{Marco analítico}

Actualmente existe una extendida preocupación, reflejada en la discusión académica iniciada hacia fines de la década del setenta 1,2,3, $4,5,6,7,8$, respecto a la escasa articulación entre investigación y políticas de salud. Más recientemente, adquiere relevancia el debate en el marco latinoamericano 9,10,11,12 y su relación con las ciencias sociales 13,14 .

Asimismo, se han producido diferentes estrategias de transferencia de conocimientos. Landry 15 distingue cuatro modelos teóricos en virtud del modo en que la investigación es producida y utilizada. Estos modelos son utilizados como base para explicar los factores que impactan en el grado de coordinación entre investigadores y tomadores de decisiones. El modelo de "impulso científico" consiste en la provisión de avances sobre la investigación por parte de los investigadores hacia los gestores. El supuesto subyacente es que la sola entrega de resultados generará tal interés por parte de los tomadores de decisión, que será sucedida por el uso de la investigación para resolver los problemas de la gestión. El segundo modelo, "tracción de demanda", supone la existencia de mecanismos de coordinación explícitos entre académicos y tomadores de decisión en una lógica contractual. En este caso, se presume que la influencia de los que toman decisiones sobre los temas de investigación promoverán su utilización y aplicación a sus problemas de gestión. En tercer lugar, "el modelo de diseminación” promueve el desarrollo de mecanismos de difusión de la información. Por último, en el "modelo de interacción" la transferencia de conocimientos se garantiza en la medida que existan distintos espacios de interacción entre los potenciales usuarios de la investigación y los investigadores.

Un rasgo importante de estos cuatro modelos es la sobrevaloración del uso instrumental de la investigación y la débil comprensión de la interacción como proceso en donde participan actores con lógicas e intereses diferentes.

La noción de "dos comunidades" utilizada por Lomas 16 resulta fecunda para explorar la naturaleza de esta relación, reconociendo la existencia de hiatos en las relaciones entre investigadores y tomadores de decisión, como resultado de su pertenencia a diferentes comunidades con lenguajes y métodos de comunicación diferentes. Como respuesta, se enfatiza la necesidad de establecer vínculos e intercambios que permitan generar una articulación como estrategia para aumentar la apropiación de la investigación y utilización de los hallazgos 17,18,19.

Denis \& Lomas 20, bajo la noción de "investigación colaborativa”, enfatizan la importancia del proceso de interacción como un fin en sí mismo, valorado por su capacidad de generar espacios de construcción colectiva de "reglas" y normas de acción que mejoran la articulación entre la investigación y el proceso de formulación e implementación de políticas de salud.

Es posible destacar el abordaje transdisciplinario como aplicación particular del "modelo de interacción", consideradas las interfases entre investigadores y tomadores de decisiones como condición necesaria para trabajar tanto sobre las brechas que se presentan en la relación, como en la misma producción de conocimiento. Desde esta perspectiva, Hufty (Hufty M. Comunicación personal; 2005) considera que el proceso de investigación adquiere un carácter abierto y estratégico, en donde investigadores y tomadores de decisiones son concebidos como actores, con pertenencias, intereses, valores y recursos diferentes.

Denis \& Lomas 20 refiriéndose a la investigación colaborativa señalan cuatro aspectos vitales que hacen al proceso de interacción. Se considera la necesidad de establecer un serio compromiso por parte de investigadores y tomadores de decisiones, dada la inversión adicional de tiempo y otros recursos de estos proyectos. Las interacciones informales son un ingrediente esencial del éxito del proceso de colaboración, promoviendo la clarificación de puntos de vista, el intercambio de opiniones, expectativas y objetivos, como condición para facilitar confianza y la utilización de mecanismos de negociación.

Un tercer aspecto se refiere a la necesidad de garantizar la conducción del proceso, con un liderazgo que promueve el compromiso de los respectivos equipos de investigación y gestión. Por último, la administración de la incertidumbre constituye una parte intrínseca de un proceso con incógnitas asociadas a las implicancias posibles y sus beneficios esperados.

\section{Características del objeto de investigación}

El SPS es un programa implementado desde el Ministerio de Salud de la Provincia de Buenos Aires. Su objetivo consiste en reformar el modelo de atención hacia un sistema que garantice el acceso y la continuidad de la provisión de los servicios 21,22 . 
El financiamiento del SPS proviene de fondos públicos y está destinado a la población carente de cobertura formal y sin capacidad económica para adquirirla. Si bien la población objetivo total es de 2,5 millones de personas, se definió un proceso de implementación gradual de cobertura. La presente etapa dirigida a población materno infantil (Seguro Público de Salud Materno Infantil-SPSMI) se inició en el 2004, correspondiente al proceso de implantación actual, y es el objeto de estudio de la pre-sente investigación. La incorporación de los beneficiarios del Plan Vida comenzó por las embarazadas, nodrizas y niños menores de 6 años.

A pesar de la persistencia de los objetivos planteados originalmente, como corolario del aprendizaje derivado de la implementación de la política se han ido introduciendo cambios en el diseño inicial, que serán comentados más adelante.

\section{Metodología y descripción del proceso de interacción}

El análisis de la evolución de las modalidades de interacción entre el EI y EG se basa en el reconocimiento de las características de diferentes etapas comprometidas durante la investigación en curso y sus implicancias más relevantes en cuanto al proceso de investigación y el proceso político de implementación del SPSMI.

El análisis de la primera etapa, dada por la producción del protocolo, reconoce las interfases de interacción entre EI y EG y el contenido de sus actividades, revalorizando los "productos" resultantes de esta etapa. En el análisis del desarrollo de las actividades de investigación, la segunda etapa, se destacan las innovaciones en la estrategia de interacción entre EI y EG, identificando sus implicancias en dimensiones asociadas con el proceso de investigación y con aspectos sustantivos del contenido de la política de salud analizada.

La metodología utilizada para el análisis de las actividades realizadas y la dinámica de la interacción entre EI y EG se ha basado en la aplicación del mapeo de alcance 23 , información originada en talleres efectuados con la participación del EI y el EG y entrevistas individuales realizadas a diferentes miembros del EI y el EG. El análisis de la evolución y las innovaciones producidas en la investigación y el proceso político se basó en fuentes secundarias, tales como el registro documental de los productos intermedios del proceso de investigación y reportes de gestión del SPS.

\section{Descripción de las fases del proceso \\ de investigación}

En la primera fase se propuso un esquema de trabajo que permitiera producir información útil, diseñar un proyecto de investigación que colaborara en la evaluación de la implementación de la fase materno infantil del SPS. La segunda fase ha buscado desarrollar una instancia de colaboración y transferencia metodológica entre investigadores y tomadores de decisión, permitiendo considerar la constitución del EG en objeto y sujeto de la evaluación.

\section{- La producción del protocolo}

Para el diseño del protocolo de investigación se desarrollaron una serie de actividades exploratorias de relevamiento y análisis de información que sirvieron como insumo para la definición de la estructura del proyecto: (i) las preguntas de investigación y sus objetivos, (ii) el marco conceptual; y (iii) la metodología a ser utilizada.

Estas actividades consistieron en: (a) un análisis institucional de las relaciones construidas por el EG en el interior de la Agencia del Seguro y con otros actores de la estructura del Estado y de la sociedad civil; (b) el análisis de la estructura de la base de beneficiarios del SPSMI; (c) el análisis de la información disponible sobre posible conformación de una red de servicios para la atención de los beneficiarios del Seguro en cada municipio; y (d) la elaboración de una encuesta dirigida al revelamiento de condiciones de acceso, utilización, percepción de la atención recibida por parte de la población a incorporar al SPSMI.

La discusión de las preguntas de investigación junto con los tomadores de decisiones permitió construir un marco interpretativo común sobre los problemas de la política en cuestión y ubicar como ejes centrales de la investigación aquellos aspectos más relevantes para la gestión del SPS.

Los intercambios ocurridos en el contexto de las actividades de diseño conceptual y metodológico enriquecieron y multiplicaron el proceso de retroalimentación iniciado. Bajo esta interacción se logró definir la metodología, los instrumentos de recolección y el marco interpretativo, en función de la naturaleza de la política estudiada y de las necesidades de la gestión. La transferencia de conocimientos vinculados a los marcos conceptuales y los abordajes metodológicos utilizados constituyeron un aspecto central para que el EG se erija en sujeto del análisis. 
La modalidad de construcción participativa del protocolo estableció una estrategia que contribuyó a la consolidación de un producto orientado por las necesidades de la gestión, la diseminación temprana y reiterada de los hallazgos, promoviendo el desencadenamiento de las etapas iniciales de la utilización de la investigación por parte del EG - siguiendo a Kothari et al. 17 la utilización es entendida aquí en términos de un proceso gradual que se inicia con la recepción y el procesamiento de la información y culmina con la aplicación de los hallazgos.

Tanto los productos derivados del desarrollo del plan de actividades, como el proceso de interacción iniciado en ese marco, se constituyeron en insumos básicos para la construcción del protocolo. En la Tabla 1 se señalan las actividades, identificando las interfases entre el EI y el EG, a través de las cuales se formularon las preguntas de investigación, y el resto de los componentes del protocolo (objetivos, marco conceptual, metodología). Estas instancias permitieron el consenso y la puesta en común de las lógicas propias de cada equipo, la construcción de un lenguaje común y la clarificación de los propósitos y valores de cada parte.

El protocolo de investigación consiste en la realización de un estudio transversal que se propone evaluar los resultados de la intervención mediante la comparación del desempeño de los servicios de primer nivel de atención, antes y después de la implantación del SPSMI. El plan de análisis se sustenta en el estudio de la relación entre los resultados del desempeño de los servicios en ocho municipios y el desarrollo de las capacidades institucionales locales, como factor condicionante. Este diseño se apo- ya sobre la hipótesis, según la cual el proceso de implementación del SPSMI mejorará la calidad de la atención médica ofrecida por los servicios de salud, en la medida que se genere un proceso de fortalecimiento institucional del conjunto de las instancias y agencias/actores involucrados en el financiamiento y organización de los servicios bajo análisis.

\section{La investigación actualmente}

En la producción y cambios producidos durante la investigación en marcha se analizan las implicancias correspondientes a: (i) aspectos conceptuales y metodológicos; y (ii) la política de salud en cuestión.

La descripción de los cambios en la investigación comprende el análisis de las transformaciones en el marco conceptual y metodológico y las modalidades de articulación entre el EG y el EI utilizados.

Las innovaciones conceptuales se refieren a la reformulación de las categorías de gobernanza, buscando una perspectiva analítica en la utilización de las dimensiones de actores, puntos nodales, normas y procesos, para describir las interfases sociales y políticas implicadas en el análisis (Hufty M. Comunicación personal; 2005). La gobernanza es considerada como una variable intermedia, condicionada por factores sociales e institucionales y condicionante del desempeño de los servicios. Al mismo tiempo, la gobernanza integra el estudio de las interfases sociales, el proceso político y decisorio en los diferentes niveles de análisis, abarcando el nivel provincial, el municipal y las condiciones locales asociadas con el funcionamiento de los servicios de atención primaria.

Construcción del protocolo.

\begin{tabular}{|c|c|c|}
\hline Actividades & Interfases & Productos protocolo \\
\hline Análisis institucional & Entrevistas y talleres con el EG & $\begin{array}{l}\text { Preguntas de investigación: ¿Cómo generar procesos } \\
\text { de reforma sustentables? }\end{array}$ \\
\hline Análisis del diseño del SPSMI & Análisis de instrumentos de gestión & $\begin{array}{l}\text { Preguntas de investigación: ¿Cuál es el nivel de desarrollo } \\
\text { y evolución de las capacidades institucionales locales en los } \\
\text { ochos municipios estudiados? }\end{array}$ \\
\hline Encuesta exploratoria & Diseño y análisis de la encuesta & $\begin{array}{l}\text { Preguntas de investigación: ¿Cuáles son los factores que condicionan } \\
\text { el desempeño de los servicios de primer nivel municipales? } \\
\text { Metodología: abordaje cuanti-cualitativo mediante el relevamiento } \\
\text { de fuentes primarias }\end{array}$ \\
\hline
\end{tabular}

EG = equipo de gestión; SPSMI = Seguro Público de Salud Materno Infantil. 
De esta forma, el nuevo marco conceptual permite abordar un proceso de reforma de la cobertura y organización de los servicios que se modifica como objeto de análisis. La incorporación y revalorización de los aspectos políticos y estratégicos de las nuevas características del SPS requirieron de la utilización de nuevas dimensiones conceptuales que den cuenta de tales innovaciones.

En segundo lugar, durante el proceso de investigación, el modelo de interacción entre el EI y el EG adquiere características diferentes. Se profundiza con la apropiación de la investigación por parte del EG y con un mejor conocimiento del SPS y su contexto por parte del EI. Nuevas interfases comprendidas por la mayor participación e involucramiento del EG en las actividades de recolección de información, en la redefinición de las mismas en función de cambios en las condiciones contextuales y políticas del proceso de implementación del SPS y la inclusión de nuevos actores locales en proceso de investigación representaron un soporte de trabajo superador del modelo previo.

Algunos hechos que señalan el progreso del involucramiento y apropiación por parte del EG son: la mayor presencia de este actor en actividades de la investigación, el impulso del EG para la organización de nuevas interfases, la designación de recursos humanos del SPS para el acompañamiento de la investigación y el aliento y apoyo hacia la generación de un fuerte involucramiento de los actores locales en las actividades de recolección y análisis de la información.

Este proceso de apropiación de la investigación por el EG se da ligado a la utilización de la investigación desde distintas dimensiones: instrumental, conceptual y simbólica. En primer lugar, se utilizaron los hallazgos de la investigación relativos a los datos producidos en la primera fase del protocolo sobre desempeño y gobernanza para sistematizar y confirmar la percepción del EG sobre el estado de situación de los ocho casos. En términos conceptuales el estudio ha aportado al EG una herramienta para repensar el monitoreo de las distintas estrategias de intervención, tanto relacionadas a la gestión política como técnica del SPS. En este sentido es interesante señalar que el EG ha definido como objeto de las innovaciones del SPS a los ocho municipios que forman parte de la investigación, en función de la posibilidad de monitorear el impacto de las intervenciones propuestas. Por otra parte, las interfases con los equipos de gestión municipales promueven la problematización de la gestión local y el diseño de estrategias de intervención con la par- ticipación de los actores locales. En términos simbólicos la investigación ha sido utilizada por el EG para aumentar su legitimidad frente a distintos actores. Asimismo, son explotados los efectos sobre el aumento de la visibilidad política del proyecto en diferentes contextos. Tanto la visibilidad del proyecto político, como el prestigio vinculado a su articulación con ámbitos académicos, otorgan al EG una mayor legitimidad en la implementación de las estrategias y la definición de nuevas normas de funcionamiento del sistema de servicios de salud.

Una profundización del entendimiento mutuo y un mayor conocimiento del terreno han modificado la orientación de las preguntas de investigación. Mientras las preguntas de investigación del protocolo inicial estaban asociadas a un esquema tradicional de evaluación, comparando las condiciones previas y posteriores a la implementación del SPS, las nuevas preguntas están orientadas a un análisis situacional, incluyendo la perspectiva e interés de los actores sociales y locales involucrados en cada uno de los estudios de casos.

En tercer lugar, los cambios metodológicos de la investigación se corresponden con las mismas exigencias del desarrollo de nuevas categorías conceptuales, condiciones y procesos en la interacción entre el EI y el EG. Al mismo tiempo, los cambios metodológicos representan deslizamientos que operan para alcanzar los nuevos desafíos de un proceso de investigación abierto a los procesos políticos relacionados, como objetos y sujetos de análisis.

Considerando los aportes de Martínez-Ravanal (Martínez-Ravanal V. Comunicación personal; 2005), los cambios metodológicos de la investigación pueden visualizarse como un deslizamiento desde una estructura inicial (centrada en un diseño cuasi experimental clásico), hacia un esquema más abierto y estratégico. Desde esta perspectiva se sugiere que el eje reflexivo se ha ido aproximando a la lógica de producción social o implementación, adquiriendo cada vez más las características de un diseño multimétodo.

El diseño multimétodo se edifica sobre seis dimensiones metodológicas que permiten caracterizar el diseño metodológico del proyecto, y a su vez entender los cambios que se han producido. A continuación se señalan los rasgos más importantes de tales transformaciones.

1. Desde un análisis sincrónico (estudio estático comparativo) a uno diacrónico: este cambio de dirección se relaciona tanto con la necesidad de flexibilizar el diseño en función del proceso de implementación del SPS, como por la visualización de la riqueza de incorporar un 
análisis de procesos. A raíz de esta transformación el diseño transversal se reconvierte en un estudio longitudinal y se incorporan técnicas de observación participante en distintas etapas de la investigación, con el fin de dar cuenta de los hallazgos en el marco de los procesos que los han generado;

2. De un análisis extensivo hacia uno intensivo: la comprensión del peso de la idiosincrasia de los espacios locales en el impacto del SPS a nivel municipal propició el rediseño del estudio de la implantación del SPS como estudio de casos;

3. De un estudio deductivo a otro inductivo: esto se relaciona con el proceso de interacción entre teoría y práctica donde el marco conceptual se va enriqueciendo y modificando en contacto con la riqueza que aporta el trabajo en terreno;

4. Desde la búsqueda de la objetividad hacia la ponderación de la subjetividad de los actores: si bien desde el inicio se previó la articulación de técnicas y fuentes de información, este abordaje estaba más centrado en el análisis del desempeño del SPS que en la comprensión de las distintas perspectivas y expectativas de los actores involucrados;

5. Desde una concepción de neutralidad de la investigación hacia el reconocimiento y análisis de la reactividad de la investigación sobre el proceso analizado. La participación de actores locales en actividades de recolección y análisis de la información resultó en una estrategia con impacto favorable en cuanto a la receptividad de los mismos, para evaluar el proceso de implementación del SPS desde diferentes perspectivas sociales y políticas;

6. Desde una metodología basada en el análisis, hacia una perspectiva de síntesis: esta orientación responde a la necesidad de comprender el impacto del SPSMI más allá de sus objetivos explícitos y formales, construyendo insumos y productos útiles para la toma de decisiones en la gestión de los servicios.

Esta reorientación ha originado un cambio en el modelo inicial de actividades con una lógica secuencial y cerrada (Figura 1), vinculada con el prototipo de investigación tradicional hacia un modelo abierto al contexto y con mayor retroalimentación y desarrollo estratégico de cada una de las actividades de la investigación (Figura 2).

\section{Cambios del SPS}

Con el propósito de revalorizar los cambios producidos en el SPS se presentan los rasgos sobresalientes de su diseño original, formulado inicialmente entre los años 1999 y 2000 y las transformaciones experimentadas durante los años 2004 y 2005.

Siguiendo la perspectiva de pluralismo estructurado 24 , el SPS fue concebido como una estrategia de reforma del sistema de salud, basado en innovaciones institucionales en la función del aseguramiento y los mecanismos de transferencia de recursos hacia la provisión de los servicios.

En cuanto al aseguramiento, el SPS explicita sus tres componentes centrales: (i) la población cubierta: definida en términos de la población sin cobertura formal, ni capacidad económica para adquirir un seguro privado; (ii) el "paquete de servicios": definidos como los servicios del primer nivel de atención médicos, bioquímicos, odontológicos y provisión de medicamentos; y (iii) los recursos financieros asignados a esta cobertura, resultantes de un realización del presupuesto capitada de las prestaciones cubiertas.

En relación a las innovaciones en las transferencias de recursos hacia la provisión se destacan los siguientes puntos: (i) la aplicación de contratos privados entre el SPS y la provisión directa de los servicios de primer nivel de atención, como organización a cargo de la "función de compra"; (ii) la introducción de "nuevos actores" en la organización del sistema de servicios, a través de la incorporación de las instituciones representativas de los profesionales como contratantes intermediarios entre el SPS y los profesionales individuales; y (iii) la innovación de las modalidades de transferencia de recursos, incorporando un sistema de "pago capitado" a los médicos y por prestación de servicios bioquímicos y odontológicos.

Los cambios producidos durante el período 2004-2005 se presentan como resultados de la dinámica propia del proceso político y como consecuencia de las nuevas interfases entre el EG y EI durante la producción del proceso de investigación. Las vinculaciones entre cambios en el proceso político y el proceso de investigación se explican por el desarrollo de nuevas modalidades de articulación asociadas con el análisis participativo de los resultados preliminares del proceso de implementación del SPS. Dichos resultados corresponden a las actividades de recopilación de datos de fuentes primarias, mediante la aplicación de encuestas y entrevistas de grupos focales con beneficiarios y trabajadoras vecinales del Plan Vida, con el fin de indagar sobre las dimensiones de utilización, accesibilidad, capacidad de respuesta y fuente regular de atención.

Considerando que la información producida por la investigación tiene relación directa 
con las necesidades explicitadas por el EG, las interfases que integran tomadores de decisiones e investigadores han asumido un rol institucional de evaluación del proceso de implantación del SPS con la inclusión y participación de los actores a cargo de la gestión municipal de los servicios de salud. De esta forma, la interrelación entre el proceso de gestión y de la investigación ha fortalecido una actitud activa del EG, al ponderar la utilidad de los resultados y utilizar los mismos en el proceso político situacional de implantación del SPSMI.

A continuación se presentan los principales cambios producidos en el SPS y su proceso político: (i) percepción de las limitaciones de la focalización restringida a la población materno infantil y la exploración de la ampliación etárea de los niños cubiertos y la incorporación de adolescentes mujeres de 14 a 19 años; (ii) cambios en la oferta de los servicios, incorporando a "obstétricas" y enfermeras comunitarias como recursos humanos integrantes del "equipo de salud". La discusión de este punto ha promovido el desarrollo de "equipos de salud" como condición de la provisión de los servicios para lograr garantizar la integridad de la atención; (iii) mayor integración del proceso de implementación del SPS en función de las características idiosincrásicas de los servicios de cada escenario local; y (iv) desarrollo de estrategias de integración del modelo de atención médica con el área de políticas sociales y con otros actores locales involucrados en los mecanismos de articulación entre la población beneficiaria y los servicios. Estas innovaciones refuerzan un proceso político definido por nuevos intereses y posicionamientos con respecto a las políticas públicas. Los rasgos más relevantes de las nuevas relaciones sociales y políticas afirman la consolidación de "alianzas" entre el EG y actores sociales locales y los equipos de gestión municipal.

Estos cambios orientan una modificación de un modelo de reforma basado en la introducción de nuevos mecanismos de contratación, incentivos económicos y la introducción de nuevos actores privados, hacia un modelo basado en el desarrollo de la atención primaria con claras estrategias de integración social y del sistema de servicios.

\section{Discusión}

En esta sección se discuten los resultados sobre los cambios del proceso de interacción entre el EG y EI, y sus implicancias en la dinámica de los aspectos conceptuales, metodológicos y el
Figura 1

Modelo secuencial.

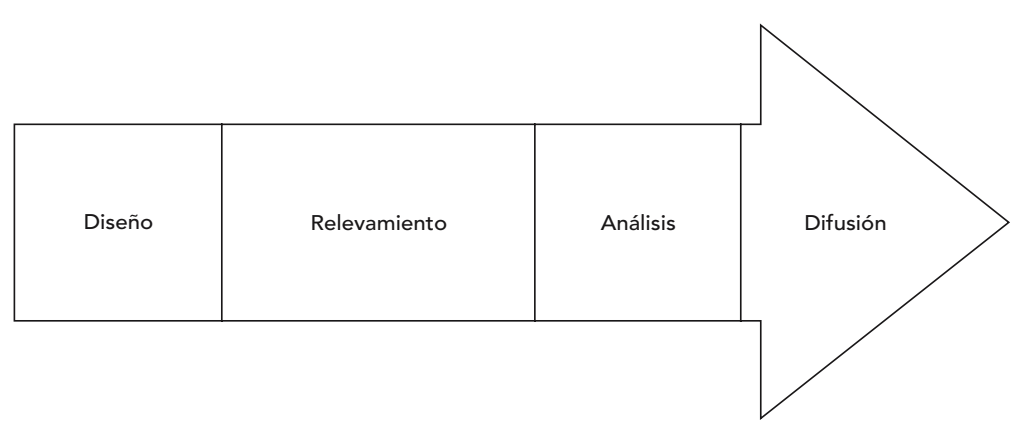

Figura 2

Modelo abierto.

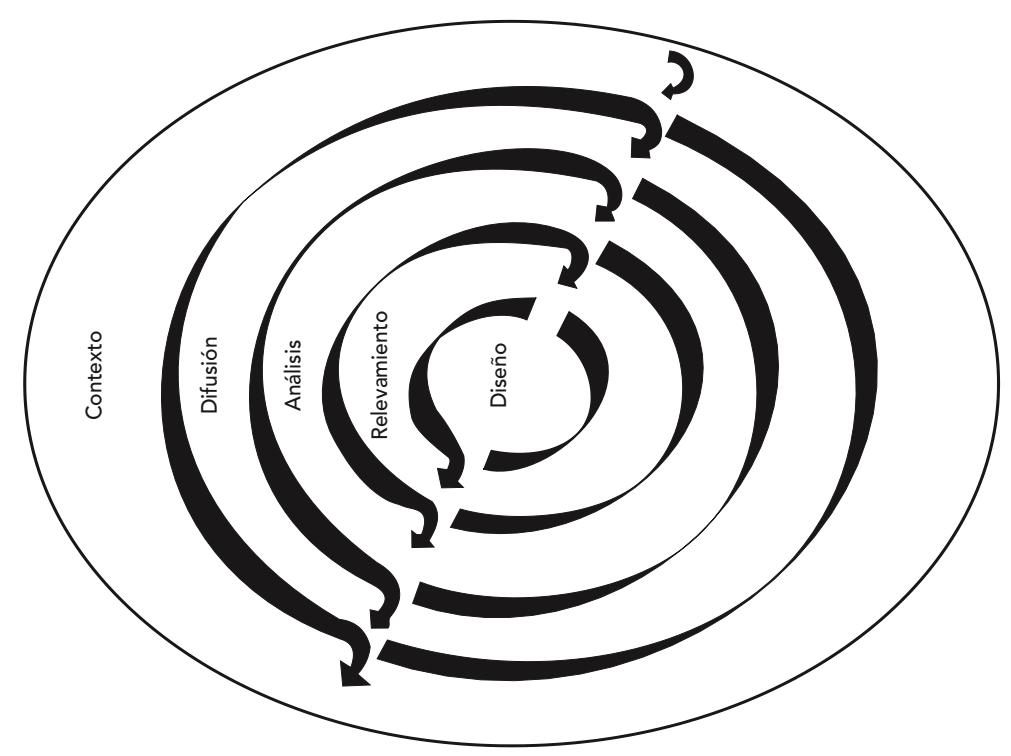

mismo proceso político como objeto de la investigación. Este análisis permite reconocer las relaciones entre las dimensiones más relevantes de este proceso y su discusión con el marco analítico.

\section{Los cambios del proceso de interacción}

Con respecto a la producción del protocolo, se generaron interfases que permitieron un intercambio de conocimientos, el autoajuste de los 
objetivos de la investigación en función de las necesidades del EG, la optimización de la diseminación y utilización temprana de la investigación y la generación de mayor receptividad por parte de los tomadores de decisiones.

La presencia de interfases resultó de vital importancia, en la generación de cambios cualitativos en la conducta del EG (desinterés-interés, rechazo-colaboración) en relación a la investigación. Por su parte, la limitación más importante de esta etapa fue la ausencia de interfases con los equipos de gestión locales. Esta situación significó el desaprovechamiento de una oportunidad que podría haber enriquecido el diseño del protocolo, mediante la incorporación de la perspectiva de los actores locales, así como la posibilidad de generar un ámbito favorable para promover su involucramiento y una futura apropiación de la investigación.

La necesidad de contar con un producto como resultado de la primera fase de la convocatoria (el diseño del protocolo) y el contexto particular de condiciones competitivas en la producción restringió el desarrollo de un proceso abierto, exploratorio y analítico sobre la política en cuestión.

Los desafíos centrales de la segunda fase se vinculan con los requerimientos producidos por un proceso abierto, en donde la producción de la investigación adquiere múltiples contactos con el proceso político analizado. En primer lugar, es necesario destacar la necesidad de manejar la tensión entre la flexibilidad y la rigurosidad metodológica. La flexibilidad es un factor que responde a la necesidad de adaptación a los cambios de las condiciones en el proceso político y el SPSMI. Al mismo tiempo, desde el lugar de los investigadores, el diseño del protocolo y los "productos" comprometidos representaron restricciones asumidas durante el propio proceso. En segundo lugar, estas innovaciones requieren por parte de EI, el desarrollo de nuevas capacidades para trabajar en un contexto más complejo, con mayor flexibilidad de adaptación a escenarios de alta incertidumbre.

En la descripción del proceso de la primera y segunda fase de la investigación es posible reconocer algunas relaciones entre las características del proceso de interacción y sus implicancias metodológicas, con innovaciones en el marco conceptual y el propio cambio del objeto de análisis y su proceso político.

Si bien en ambos momentos se procuró construir un modelo de interacción transdisciplinario, la producción del protocolo se basó en estrategias signadas por estrategias del tipo "push-pull", dirigidas a promover la transferencia de conocimientos, con el propósito de "construir puentes" que mejoren la comunicación y el entendimiento entre ambas comunidades. El énfasis de esta etapa es la producción participativa de actividades específicamente diseñadas para la obtención de "productos" correspondientes a los componentes del protocolo de investigación.

En cambio, en la segunda fase prevaleció un modelo de interacción entre los integrantes de ambos equipos, fortaleciendo el mutuo reconocimiento, tanto en actores de un proceso abierto, como en las implicancias reconocidas en el desarrollo de la investigación y el proceso político. Esta fase permite reconocer la maduración de estrategias que promueven un modelo de interacción, como variable independiente de un proceso con relaciones complejas e implicaciones que alcanzan el mismo objeto de análisis y su abordaje conceptual y metodológico.

En la Figura 3 se presentan las principales relaciones entre las dimensiones consideradas, definiendo la modalidad de interacción entre el EI y el EG como variable de cambio con implicancias sobre el marco conceptual y metodológico y sobre el proceso político que contiene el objeto de análisis.

Se puede reconocer que los cambios operados entre el momento de la producción del protocolo y la realización de la investigación se manifiestan tanto en modificaciones en las diferentes dimensiones consideradas, como en un proceso de mayor condicionamiento mutuo e intercambio entre las mismas. Este marco permite explicitar las relaciones producidas por una estrategia de interacción entre EG y EI, como generador y condicionado por innovaciones en (i) el desarrollo y aplicación del marco conceptual y (ii) el proceso político de implementación del SPSMI. Mientras la primera dimensión busca dar cuenta de los cambios del proceso de investigación, como resultado del modelo de interacción implementado, la segunda dimensión revaloriza los cambios en las intervenciones de extensión de la protección social en salud implementadas, como resultado de esta investigación. Además, una articulación activa y explícita entre investigadores y tomadores de decisión desafía los conceptos tradicionales de neutralidad del investigador como sujeto y actor ajeno y neutral del análisis del objeto estudiado.

Finalmente, la mutua condicionalidad de estas relaciones asume al modelo de interacción considerado como variable endógena del proceso implementado. La producción de nuevas reglas de juego que regulan el modelo de interacción es una expresión de la dinámica de este proceso. 


\section{Conclusiones}

Este artículo analiza la relación entre investigadores y tomadores de decisiones en un proyecto de investigación (actualmente en marcha), señalando los principales cambios en las modalidades de articulación durante los momentos de producción del protocolo y los primeros diez meses de desarrollo del proyecto. La información presentada permite reconocer que un abordaje transdisciplinario representa un desafío que requiere un importante compromiso de las partes involucradas, ya que esta modalidad conlleva múltiples incertidumbres que atentan contra un modelo tradicional de investigación.

El abordaje transdiciplinario puede ser entendido como resultado de una construcción gradual y sostenida en el tiempo, ya que necesita de un lapso suficiente de tiempo, para lograr la sensibilización y receptividad de los tomadores de decisiones y construir y consolidar un vínculo entre ambos equipos. La construcción de una modalidad de interacción supone la construcción de interfases con capacidad de negociación y colaboración entre "comunidades” con prácticas, intereses y expectativas diferentes. El proceso de cambio que desencadena esta perspectiva requiere la generación de nuevas "reglas" que articulen la investigación con las condiciones concretas del objeto de análisis, sus transformaciones y el interés de los actores con responsabilidad en la formulación y gestión de las políticas. En este estudio de caso, estas condiciones son alcanzadas sólo recién en el proceso de producción de la investigación, una vez integrados los tomadores de decisiones en una gran diversidad de actividades. La consolidación de esta modalidad ha requerido la adaptación de la investigación a los cambios en el proceso de implementación del
Figura 3

Implicancias del proceso de interacción.

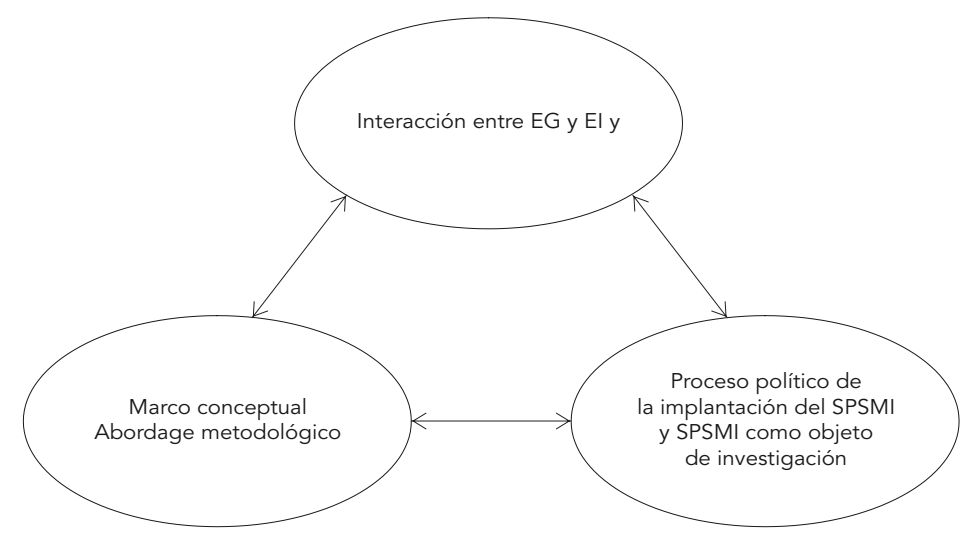

SPS y la definición de nuevas "reglas" de articulación de las actividades de investigación en instancias de gestión y coordinación del SPS con los municipios estudiados. Igualmente, una mayor integración entre EI y EG durante todo el proceso de investigación ha permitido expandir el alcance de las implicancias de este proceso de producción académica, tanto en sus herramientas conceptuales, como en el mismo objeto de análisis.

Finalmente, si bien el proceso de investigación ha logrado enlazar la investigación y sus resultados preliminares en función de los intereses del EG, el análisis presentado corresponde a una etapa de un proceso inacabado, en donde la incertidumbre del contexto institucional vigente impide predecir la evolución de la dinámica política en consideración.

\section{Resumen}

Este artículo analiza el proceso de interacción entre el equipo de investigación del Instituto de la Salud Juan Lazarte y el equipo de gestión del Seguro Público de Salud (SPS) de la Provincia de Buenos Aires, Argentina, durante el diseño y desarrollo de la investigación Análisis de las Capacidades Institucionales y Evaluación de De-sempeño del SPS. Dada la existencia de distintos espacios de interacción entre el equipo de gestión del SPS y el equipo de investigación, su relación es caracterizada como una aplicación del "modelo de interacción", desde una perspectiva transdiciplinaria.
Este abordaje promueve interfases que permiten el desarrollo de procesos de negociación y colaboración entre las "comunidades" científicas y políticas. La aplicación de este modelo de interacción desencadenó cambios en el marco conceptual y metodológico, y sobre aspectossustantivos del proceso de implementación del Seguro Público de Salud Materno Infantil.

Seguro de Salud; Interaccion Social; Gestión en Salud 


\section{Colaboradores}

E. Báscolo fue responsable por el desarrollo con-ceptual, las implicancias del modelo de interacción y los cambios en el proceso de investigación. N. Yavich participó de la discusión y colaboración integral en la redacción del documento. A. S. de León realizó el análisis de los cambios del Seguro Público de la Provincia de Buenos Aires.

\section{Referencias}

1. Caplan N. The two-communities theory and knowledge utilization. Am Behav Sci 1979; 22 Suppl 3:459-70.

2. Weiss $\mathrm{CH}$. The many meanings of research utilization. Public Adm Rev 1979; 39:426-31.

3. Backer TE. Knowledge utilization: the third wave. Knowledge: Creation, Diffusion, Utilization 1991; 12:225-40.

4. Estabrooks C. The conceptual structure of research utilization. Res Nurs Health 1999; 22:203-16.

5. Moynihan R. Using health research in policy and practice: case studies from nine countries. Ney York: AcademyHealth/The Milbank Memorial Fund; 2004.

6. Bowen S, Zwi AB. Pathways to "evidence-informed" policy and practice: a framework for action. PLoS Med 2005; 2:e166.

7. Canadian Health Services Research Foundation. Issues in linkage and exchange between researchers and decision makers. Ottawa: Canadian Health Services Research Foundation; 1999.

8. Landry RN, Amara N, Lamari M. Utilization of social science research knowledge in Canada. Research Policy 2001; 30 Suppl 2:333-49.

9. Furtado JP. Um método construtivista para a avaliação em saúde. Ciênc Saúde Coletiva 2001; 6: 165-81.

10. Pittman P. Allied research: experimenting with structures and processes to increase the use of research in health policy. In: Global Forum for Health Research - Final Documents [CD-ROM]. Mexico DF: Global Forum for Health Research; 2004.

11. Barreto M. O conhecimento científico e tecnológico como evidência para políticas e atividades regulatórias em saúde. Ciênc Saúde Coletiva 2004; 9:329-38.

12. Frenk J. Balancing relevance and excellence: organizational responses to link research with decision making. Soc Sci Med 1992; 35:1397-404.

13. Greenberg JN, Choi T. The role of the social sciences in the health services research: an overview. In: Choi T, Greenberg JN, editors. Social science approaches to health services research. Chicago: Health Administration Press; 1992. p. 2-20.

14. Brown L. Knowledge and power: health services research as a political resource. In: Ginzberg E, editor. Health services research: key to health policy. Cambridge: Harvard University Press; 1991. p. 20-45.

\section{Agradecimientos}

Deseamos expresar nuestro reconocimiento a la Organización Panamericana de la Salud y el International Development Research Centre, cuyo respaldo posibilitó el desarrollo de esta investigación, y en especial a Eduardo Levcovitz, Soledad Urrutia, Roberto Bazzani, Patricia Pittman y Celia Almeida.
15. Landry R. Linkage and exchange as determinants of research utilization: the rationale and some international evidence. In: Issues in linkage and exchange between researchers and decision makers. Ottawa: Canadian Health Services Research Foundation; 1999. p. 18.

16. Lomas J. Improving research dissemination and uptake in the health sector: beyond the sound of one hand clapping. Ontario: Centre for Health Economics and Policy Analysis, McMaster University; 1997.

17. Kothari A, Birch S, Charles C. "Interaction" and research utilization in health policies and programs: does it work? Health Policy 2005; 71:117-25.

18. Bronfman M, Trostle J. El papel de la investigación en políticas de salud: una revisión estratégica de la literatura. In: Bronfman $\mathrm{M}$, Castro $\mathrm{R}$, organizadores. Salud, cambio social y políticas: perspectivas desde América Latina. México DF: EDAMEX; 1999.

19. Trostle J, Bronfman M, Langer A. How do researchers influence decision-makers? Case studies of Mexican policies. Health Policy Plan 1999; 14:103-14.

20. Denis JL, Lomas J. Convergent evolution: the academic and policy roots of collaborative research. J Health Serv Res Policy 2003; 8 Suppl 2:1-6.

21. Seguro Público de Salud. Manual del médico de cabecera. Buenos Aires: Ministerio de Salud, Provincia de Buenos Aires; 2003.

22. Seguro Público de Salud. Informe de gestión 2004. Buenos Aires: Ministerio de Salud, Provincia de Buenos Aires; 2005.

23. Earl S, Carden F, Smutylo T. Outcome mapping. Building learning and reflection into development programs. Ottawa: International Development Research Council; 2001.

24. Londoño JL, Frenk J. Structured pluralism: towards an innovative model for health system reform in Latin America. Health Policy 1997; 41 Suppl 1:1-36.

Recibido el 21/Nov/2005

Versión final presentada el 17/Abr/2006

Aprobado el 25/Abr/2006 